\title{
Antifungal Activity of the Essential Oil of Echinops kebericho Mesfin: An In Vitro Study
}

\author{
Tamirat Bekele Beressa $\mathbb{D}^{1,2,3}$ Serawit Deyno, ${ }^{1,2,4}$ and Paul E. Alele $\mathbb{C}^{1}$ \\ ${ }^{1}$ Department of Pharmacology and Therapeutics, Faculty of Medicine, Mbarara University of Science and Technology, \\ P.O. Box 1410, Mbarara, Uganda \\ ${ }^{2}$ Pharm-biotechnology and Traditional Medicine Center, Mbarara University of Science and Technology, Mbarara, Uganda \\ ${ }^{3}$ Department of Pharmacy, College of Medicine and Health Sciences, Ambo University, Ambo, Ethiopia \\ ${ }^{4}$ Department of Pharmacology, School of Pharmacy, College of Medicine and Health Sciences, Hawassa University, \\ Hawassa, Ethiopia
}

Correspondence should be addressed to Paul E. Alele; paulalele@must.ac.ug

Received 4 July 2020; Revised 29 October 2020; Accepted 31 October 2020; Published 12 November 2020

Academic Editor: Jenny M. Wilkinson

Copyright ( 2020 Tamirat Bekele Beressa et al. This is an open access article distributed under the Creative Commons Attribution License, which permits unrestricted use, distribution, and reproduction in any medium, provided the original work is properly cited.

\begin{abstract}
Background. Echinops kebericho is an endemic medicinal plant in Ethiopia widely used in the treatment of infectious and noninfectious diseases. Essential oils are known for their antibacterial, antifungal, antiviral, insecticidal, and antioxidant properties. This study evaluated the antifungal activity of essential oil from E. kebericho against four common pathogenic fungi and two standard strains. Methods. The essential oil was obtained by hydrodistillation. The antifungal screening was done by agar well diffusion method. Minimal inhibitory concentrations (MICs) were determined by broth microdilution. Minimal fungicidal concentrations (MFCs) were determined by subculturing fungal strains with no visible growth onto a Sabouraud dextrose agar (SDA) plate. Results. Candida albicans and Cryptococcus neoformans were highly sensitive while Aspergillus flavus did not show sensitivity up to $1 \mathrm{mg} / \mathrm{ml}$ of essential oil; MICs ranged from $0.083 \mathrm{mg} / \mathrm{ml}$ to $0.208 \mathrm{mg} / \mathrm{ml}$. Concentration and fungal species showed significant dose-dependent associations $(p<0.0001)$ with antifungal activity. The MICs of essential oil were comparable to those of the standard drug (fluconazole) against C. glabrata and C. krusei. The lowest MFC of the essential oil was observed against Candida parapsilosis $(0.145 \mathrm{mg} / \mathrm{ml})$ while the highest MFC was against Candida krusei $(0.667 \mathrm{mg} / \mathrm{ml})$. Conclusion. Echinops kebericho essential oil showed noteworthy antifungal activity against Cryptococcus neoformans, Candida albicans, and Candida glabrata and could be a potential candidate for further antifungal drug development.
\end{abstract}

\section{Background}

Fungi are eukaryotic organisms that cause a range of clinical infections including skin, hair, nail, mucosal, subcutaneous, and invasive infections. The majority of fungi cause opportunistic infections in immune-compromised people or individuals taking immune suppressive drugs $[1,2]$. These include people with HIV/ AIDS, primary immune deficiency, cancer chemotherapy, hematologic and solid organ transplantation, and prematurity and those on immune-modulatory medications [3]. More than $90 \%$ of reported fungal-associated deaths are caused by species belonging to three genera: Cryptococcus, Candida, and Aspergillus [4].

The burden of fungal infections is alarming in the world: more than 10 million people have mucosal candidiasis and more than 150 million people have serious fungal diseases, which have a major impact on their lives or are fatal [5]. Mortality associated with a fungal disease is more than 1.6 million, similar to that of tuberculosis and over 3 -fold more than malaria [6]. The major concern of fungal infections is the high rate of mortality associated with invasive fungal infections, which often exceeds $50 \%$ despite the availability of antifungal drugs [4]. This is due to the emergence of 
widespread drug resistance against antifungal agents that limits treatment options [7].

Antifungal treatment options are highly limited, as there are few chemical classes represented by existing antifungal drugs [4]. To date, there are three different classes of antifungal agents, polyenes, azoles, and echinocandins [8]. Drug toxicity is the major limitation of polyenes and the older generation of azole antifungal agents. New antifungal agents (e.g., echinocandins and second-generation triazoles) in the past decade have transformed the management of invasive mycoses. These newer antifungal agents have also important limitations such as in pharmacokinetics, drugdrug interactions, and unusual toxicities associated with long-term use [9].

Echinops kebericho is an endemic medicinal plant widely used in Ethiopia. It is used to treat different infectious and noninfectious diseases such as fever, headache, stomachache, malaria, and cough in the community [10]. Extracts and essential oils of the roots of E. kebericho have been assessed for their antimicrobial [11], anthelminthic [12], and molluscicidal activities [13].

Essential oils are known for their antibacterial, antifungal, antiviral, insecticidal, and antioxidant properties [14]. They are widely used in traditional medicine and the food industry. The main constituents of essential oils are terpenes, terpenoids, aromatic and aliphatic constituents, and some hydrocarbons, which also exhibit antimicrobial effects [15]. Studies have shown that essential oils target the cell wall membrane, thereby disturbing ATP production and $\mathrm{pH}$ homeostasis [16]. A study conducted by Ameya et al. on the crude methanol and ethanol extracts of E. kebericho demonstrated activity against clinical isolates of Candida albicans and Aspergillus flavus [11]. Though the research was done on the crude extract against the two strains, the effect on the other fungal strains is unknown, and there has been no study on the essential oil of E. kebericho. The aim of this study, therefore, was to evaluate the antifungal activity of the essential oil of E. kebericho M. against Candida albicans, Candida glabrata, Cryptococcus neoformans, and Aspergillus flavus.

\section{Methods}

2.1. Study Design and Setting. This was an in vitro experimental study. The extraction of the plant essential oil was carried out at the Pharmacognosy Laboratory at Addis Ababa University. Determination of in vitro activity was done at the Mycology Unit of the Microbiology Laboratory at Mbarara University of Science and Technology. Clinical fungal strains of C. albicans, C. glabrata, C. neoformans, and A. flavus and quality control strains, C. parapsilosis ATCC 22019 and C. krusei ATCC 6258, were used for this study.

2.2. Chemicals and Equipment. For this study, we used 0.5 McFarland Standard, dimethyl sulphoxide (DMSO), Sabouraud's dextrose agar, RPMI 1640, potato dextrose agar, Mueller-Hinton agar, glucose, and methylene blue dye. The equipment used included a digital measuring balance, vortex, mortar, and pestle; and the apparatus included beakers, conical and round-bottom flasks, inoculating loops, pipettes, 96-well microtiter plates, densitometer, Clevenger apparatus, incubator, autoclave, and dry oven.

2.3. Plant Collection and Extraction. Fresh tubers of E. kebericho were collected from areas around Addis Ababa, Ethiopia. A taxonomist identified the plant and a voucher number of $001 \mathrm{~TB} / 19$ was given and deposited at Addis Ababa University (AAU) Herbarium. The tubers were separated from the rest of the plant parts and washed. After air-drying in shade, the tuber material was pounded using a mortar and pestle to a fine powder; then $500 \mathrm{gm}$ of the powder was dissolved in water in a 1:5 (weight-by-volume) ratio in a round-bottom flask and hydrodistilled for 3 hours using a Clevenger apparatus [17]. The volume of the essential oil obtained in the receiver was measured and stored in the refrigerator until used.

\subsection{Determination of Antifungal Effect}

2.4.1. Preparation of Essential Oil for Antifungal Activity Assay. Essential oil (0.1 gram) was dissolved in $8 \mathrm{ml}$ of $1 \%$ DMSO and filled up to a total solution of $10 \mathrm{ml}$ with DMSO. A twofold dilution was prepared from this stock solution, which was filled in each well [18]. The EO of $10 \mathrm{mg} / \mathrm{ml}$ to $0.038 \mathrm{mg} / \mathrm{ml}$ concentration was used for the antifungal test.

2.4.2. Well Diffusion Assay. Well diffusion assay for Candida spp. and Cryptococcus spp. was conducted according to M44-A Standard [19, 20]. Sabouraud's dextrose agar (SDA) media for yeast and potato dextrose agar were used for the subculturing of the A. flavus [20, 21]. Mueller-Hinton agar supplemented with $2 \%$ glucose and $0.5 \mu \mathrm{g} / \mathrm{ml}$ methylene blue dye medium was used for testing of Candida spp. and Cryptococcus spp. [20]. Nonsupplemented Mueller-Hinton agar was used for testing of the A. flavus [21]. Fluconazole $(0.25 \mathrm{mg} / \mathrm{ml})$ was used as a positive control for Candida spp. and Cryptococcus spp. [22]. Amphotericin B (0.1 mg/ml) was used as a positive control for A. flavus [21]. For the negative control, 1\% DMSO was used [11]. After the inoculation of the organisms, $8 \mathrm{~mm}$ wells were created and filled with $100 \mu \mathrm{l}$ of different concentrations of essential oil. Positive control $(100 \mu \mathrm{l})$ and negative control were also added [2]. All the plates were prepared in triplicate and incubated at $35^{\circ} \mathrm{C}$ for 24 hours for Candida spp. and 48 hours for Cryptococcus spp. At the end of the incubation period, the diameter of the zone of inhibition in each of the inoculated plates was measured and the diameter of the well was subtracted to determine the antifungal activities of the essential oil $[2,11$, 18].

2.4.3. Broth Microdilution. Broth microdilution was used to determine the minimum inhibitory concentration (MIC). Sabouraud's dextrose agar (SDA) media were used to subculture yeast following the recommendation of 
international guidelines [23]; for A. flavus, potato dextrose agar (PDA) media were used $[21,24]$. The essential oil was prepared in serial dilutions in dimethyl sulphoxide (DMSO). $100 \mathrm{mg}$ of E. kebericho essential oil was measured and DMSO was added to $1 \mathrm{ml}$ total solution forming $100 \mathrm{mg} / \mathrm{ml}$. Serial dilution was made in twofold dilution $(1.56 \mathrm{mg} /$ $\mathrm{ml}-100 \mathrm{mg} / \mathrm{ml}$ ). Each serially diluted essential oil was further diluted in a ratio of $1: 50$ in RPMI media (100 $\mu$ l of stock solution was added to $4.9 \mathrm{ml}$ of the RPMI), giving dilutions in the range of 0.03125 to $2 \mathrm{mg} / \mathrm{ml} .100 \mu \mathrm{l}$ from each dilution was filled in the 96-well plates in accordance with CLSI protocol. The essential oil in the well was further diluted when the inoculum was added to the well to obtain dilutions in the range of $0.0156 \mathrm{mg} / \mathrm{ml}-1 \mathrm{mg} / \mathrm{ml}$ [23]. Fluconazole and amphotericin B were used as standard drugs for yeasts and Aspergillus, respectively. Twofold serial dilutions ranging from 0.25 to $128 \mu \mathrm{g} / \mathrm{ml}$ for fluconazole and 0.031 to $16 \mu \mathrm{g} / \mathrm{ml}$ for amphotericin $\mathrm{B}$ were used as a positive control; $1 \%$ DMSO was used as a negative control. Quality was controlled by using quality control strains: C. parapsilosis (ATCC 90018) and C. krusei (ATCC 6258) [25]. The MIC was the lowest concentration that inhibited the visible growth of the fungi.

2.4.4. Inoculum Preparation. The inoculum was prepared according to CLSI guidelines [23, 24]: C. albicans, C. glabrata, C. neoformans, C. parapsilosis ATCC 22019, and C. krusei ATCC 6258 were subcultured on SDA media, while A. flavus was subcultured on PDA media. The inoculum was prepared from 24-hour-old colonies of Candida species. Approximately $1 \mathrm{~mm}$ diameter was suspended in $5 \mathrm{ml}$ of saline and adjusted to $0.5 \mathrm{McFarland}$ Standard. For Cryptococcus, the 72-hour-old colonies were suspended in $5 \mathrm{ml}$ of saline and adjusted to $0.5 \mathrm{McFarland}$ standards. The prepared suspension was vortexed for 15 minutes and the cell density was adjusted to 0.5 McFarland standards. This suspension was mixed for 15 seconds with a vortex, diluted to $1: 50$, and further diluted to $1: 20$ with RPMI medium to obtain the twofold test inoculum. The (twofold) inoculum was diluted when the wells were inoculated [23]. For A. flavus, the spores were harvested from 7-day-old cultures on PDA. The saline was poured into the PDA slant and mixed with a sterile swab. After 10 minutes of sedimentation, the suspension was transferred to a sterile test tube and adjusted to the $0.5 \mathrm{McF}$ arland standards. This suspension was further diluted to $1: 50 \quad(100 \mu \mathrm{l}$ of 0.5 McFarland Standard was added to $4.9 \mathrm{ml}$ RPMI media); $100 \mu \mathrm{l}$ of the diluted was then added to the well [24]. The minimal fungicidal concentrations (MFCs) were determined by subculturing fungal isolates from the wells with no visible growth onto SDA plates. The inoculated plates were incubated for 3 days at $35^{\circ} \mathrm{C}$. MFC was the lowest concentration of drug that yielded a negative subculture $[23,26]$.

2.5. Statistical Analysis. The results were graphically presented as mean $\pm \mathrm{SD}$. Linear regression was used to determine the relationship between the concentration of the essential oil as an independent variable and zone of inhibition (response) as a dependent variable. Two-way analysis of variance was used to compare the effect of treatment using $\mathrm{EO}$ on the test microorganisms and to examine the difference in MIC of essential oil at different concentrations and fluconazole for each test strain. The independent variables were fungal strain and treatment (essential oil, fluconazole, and negative control). $p$ value $<0.05$ was considered statistically significant. Statistical analysis was done using GraphPad Prism 5.01 software.

2.6. Ethical Considerations. The research protocol was approved by the Research Ethics Committee (REC) of Mbarara University of Science Technology (approval number 05/01-19).

\section{Results}

The percentage yield of the essential oil was $0.18 \% \mathrm{w} / \mathrm{v}$ of dried weight.

3.1. Agar Well Diffusion Assay. In agar well diffusion, wells filled with $100 \mu \mathrm{l}$ of different concentrations of $\mathrm{EO}$ produced inhibition zones ranging from $3 \mathrm{~mm}$ to $17 \mathrm{~mm}$. The largest diameter was against $C$. neoformans and the lowest was against $C$. krusei ATCC 6258. The zone of inhibition of controls varied from $10 \pm 2 \mathrm{~mm}$ to $22 \pm 2 \mathrm{~mm}$. The lowest diameter was produced by $A$. flavus and the widest was produced by $C$. albicans (Table 1 ). Two-way analysis of variance showed that both concentration and fungal species had significant effects $(p<0.0001)$ on the zone of inhibition (Table 1); for concentration, $F=39.08$, $\mathrm{df}=9, p<0.0001$; and for fungal species, $F=32.41, \mathrm{df}=4, p<0.0001$.

3.2. Minimum Inhibitory Concentration. The evaluation of the MIC of E. kebericho essential oil showed notable activity against all fungal strains tested except for A. flavus. Candida parapsilosis (ATCC 22019) and Candida krusei (ATCC 6258) which were used as quality controls had MICs in the recommended ranges. The MIC ranged from $0.083 \mathrm{mg} / \mathrm{ml}$ to $0.208 \mathrm{mg} / \mathrm{ml}$. Candida albicans and Cryptococcus neoformans were highly sensitive, showing comparable activity to C. parapsilosis. Aspergillus flavus did not show sensitivity up to $1 \mathrm{mg} / \mathrm{ml}$ of the essential oil. There were no significant differences in MIC of essential oil and fluconazole against yeast $(p$ value $>0.05)$. The essential oil showed activity comparable to that of the standard drug (fluconazole) against C. glabrata and C. krusei (Figure 1).

All the strains tested were susceptible to the standard drug (fluconazole for Candida species and Cryptococcus neoformans; amphotericin B for A. flavus). Linear regression showed that there was a positive relationship between concentration and zone of inhibition (Figure 2). The slopes for all, except $A$. flavus, were positive. The essential oil did not show a zone of inhibition against the isolate of $A$. flavus. The negative control (DMSO) did not show a zone of inhibition. 
TABLE 1: Antifungal activity of Echinops kebericho EO against pathogenic fungi using the agar well diffusion method. ND, Not determined; -, No zone of inhibition observed. ${ }^{* * *}, p<0.001$ compared with $C$. krusei; ${ }^{* *}, p<0.01$ compared with C. krusei; ${ }^{*}, p<0.05$ compared with C. krusei; ${ }^{\# \# \#, ~} p<0.001$ compared with C. krusei; ${ }^{\#}, p<0.01$ compared with C. krusei; ${ }^{\#}, p<0.05$ compared with C. krusei. Values are the means of the zone of inhibition $\pm \mathrm{SD}$ of three replicates $(N=3$ replications).

\begin{tabular}{|c|c|c|c|c|c|c|}
\hline \multirow[b]{2}{*}{ Concentration $(\mathrm{mg} / \mathrm{ml})$} & \multicolumn{6}{|c|}{ Zones of inhibition $(\mathrm{mm})($ mean $\pm \mathrm{SD})$} \\
\hline & $\begin{array}{l}\text { Aspergillus } \\
\text { flavus }\end{array}$ & $\begin{array}{c}\text { Candida } \\
\text { krusei }\end{array}$ & $\begin{array}{l}\text { Candida } \\
\text { glabrata }\end{array}$ & $\begin{array}{c}\text { Candida } \\
\text { parapsilosis }\end{array}$ & $\begin{array}{c}\text { Cryptococcus } \\
\text { neoformans }\end{array}$ & Candida albicans \\
\hline 10 & - & $10 \pm 2$ & $13 \pm 1$ & $15 \pm 4$ & $17 \pm 2^{* *}$ & $14 \pm 3$ \\
\hline 5 & - & $9 \pm 1$ & $12 \pm 1$ & $13 \pm 5$ & $17 \pm 2^{* * *}$ & $14 \pm 3$ \\
\hline 2.5 & - & $8 \pm 1$ & $11 \pm 2$ & $12 \pm 3$ & $15 \pm 2^{* *}$ & $14 \pm 3^{\#}$ \\
\hline 1.25 & - & $8 \pm 1$ & $10 \pm 2$ & $12 \pm 5$ & $13 \pm 2$ & $12 \pm 2$ \\
\hline 0.625 & - & $6 \pm 1$ & $11 \pm 3$ & $10 \pm 4$ & $9 \pm 2$ & $12 \pm 3^{\#}$ \\
\hline 0.313 & - & $5 \pm 1$ & $10 \pm 2$ & $8 \pm 4$ & $11 \pm 1^{*}$ & $13 \pm 3^{\# \# \#}$ \\
\hline 0.156 & - & $3 \pm 1$ & $8 \pm 1$ & $8 \pm 4$ & $7 \pm 4$ & $12 \pm 4^{\# \# \#}$ \\
\hline 0.078 & - & $3 \pm 1$ & $7 \pm 1$ & $6 \pm 3$ & $6 \pm 2$ & $10 \pm 1^{\# \#}$ \\
\hline 0.039 & - & $3 \pm 1$ & $6 \pm 0$ & $5 \pm 2$ & $5 \pm 2$ & $9 \pm 2^{\#}$ \\
\hline Fluconazole & ND & $12 \pm 3$ & $17 \pm 3$ & $22 \pm 2$ & $19 \pm 3^{* *}$ & $22 \pm 1^{\# \# \#}$ \\
\hline Amphotericin B & $15 \pm 1$ & ND & ND & ND & ND & ND \\
\hline DMSO & - & - & - & - & - & - \\
\hline
\end{tabular}

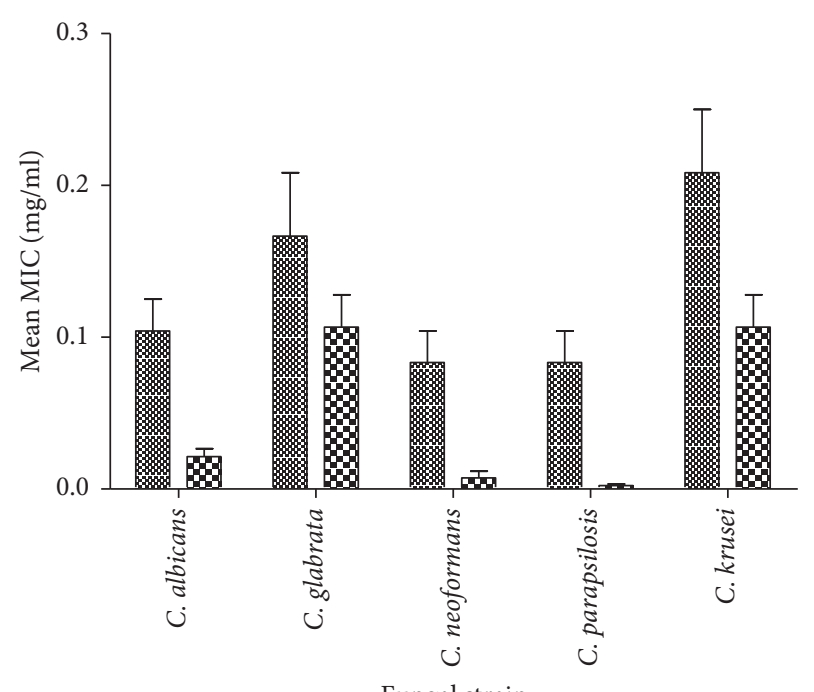

Fungal strain

MIC of essential oil

B MIC of fluconazole

FIGURE 1: Comparison of the minimum inhibitory concentration of essential oil and fluconazole against fungal strains. Values are the means of the minimum inhibitory concentrations \pm SD of three replicates ( $N=3$ replications).

3.3. Minimum Fungicidal Concentration. The essential oil showed the lowest MFC against C. parapsilosis and the highest MFC against C. krusei (Figure 3). The essential oil, however, did not show a fungicidal effect against $A$. flavus and $C$. albicans in the range of concentrations tested.

\section{Discussion}

The present study investigated the antifungal activity of the essential oil of E. kebericho on highly pathogenic invasive fungal strains, C. albicans, C. glabrata, C. neoformans, and A. flavus and quality control strains $C$. parapsilosis and C. krusei. The study demonstrated noteworthy antifungal

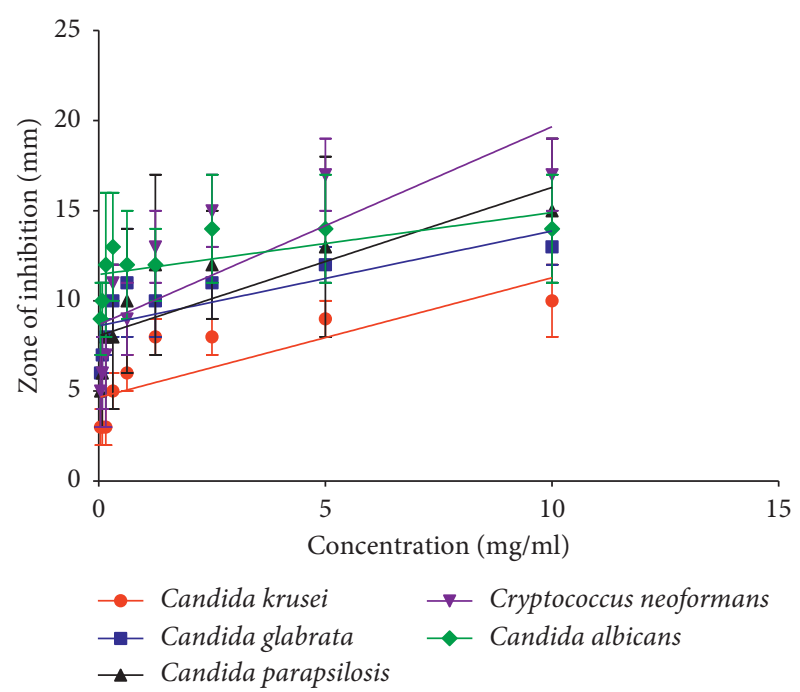

Figure 2: Linear regression showing the relationship between concentration and the zone of inhibition of essential oil for each strain. The straight line shows the line of best fit. The slope was significantly nonzero for all the strains, except $C$. albicans ( $p=0.0091$ for C. krusei; $p=0.0193$ for C. glabrata; $p=0.0075$ for C. parapsilosis; $p=0.0117$ for C. neoformans; and $p=0.0626$ for C. albicans). The $R^{2}$ for C. krusei was 0.6452 , for $C$. glabrata was 0.5660 , for C. parapsilosis was 0.6633 , for C. neoformans was 0.6210 , and for C. albicans was 0.4115 . Values shown are means $\pm \mathrm{SD} ; n=3$ replicates.

activity of the essential oil of E. kebericho against the fungal strains: $C$. neoformans was highly sensitive to the essential oil of E. kebericho, followed by C. albicans, while C. glabrata was moderately sensitive. Aspergillus flavus, however, was not sensitive at all the tested concentrations. The zone of inhibition diameter decreased as the concentration of the essential oil decreased, showing a dose-dependent effect. The dose-dependent effect was similar to a previous study conducted with peppermint oil against fungal strains [27]. Fungicidal effects were observed against C. glabrata, 


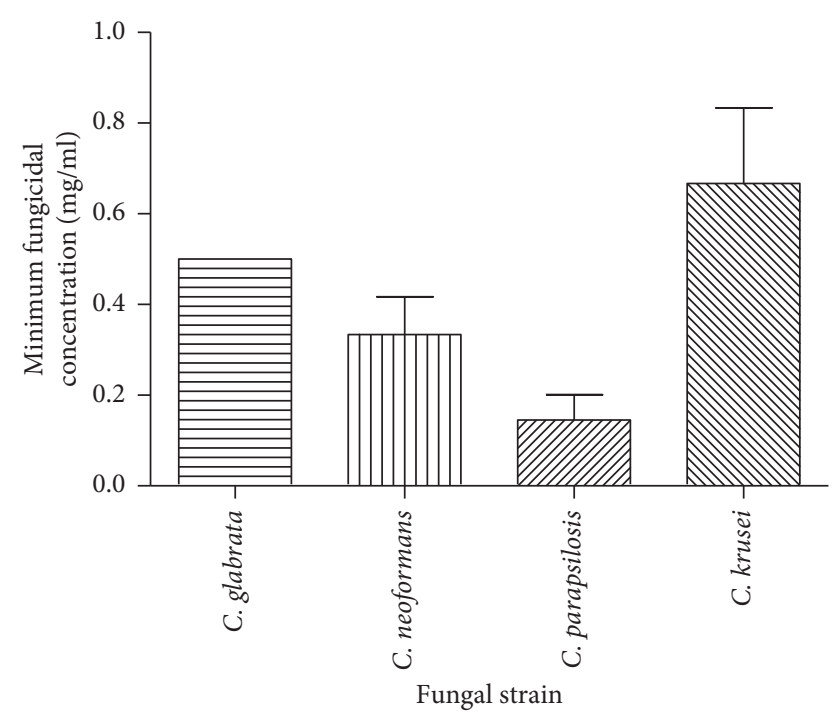

Figure 3: Minimum fungicidal concentrations of essential oil from E. kebericho against tested fungal strains. Values are the means of the minimum fungicidal concentrations \pm SD of three replicates ( $N=3$ replications). The $\mathrm{SD}$ for $C$. glabrata was zero.

C. neoformans, C. krusei ATCC 6258, and C. parapsilosis ATCC 22019.

Screening of medicinal plants for antifungal activity is important for novel drug discovery [28]. In many countries, fungal diseases have been treated using herbal remedies as many pathogenic fungi are becoming commonly resistant to numerous conventional antifungal drugs [29]. This study is the first of its kind to investigate the antifungal activity of essential oil from E. kebericho, although a previous study showed noteworthy antifungal activity using methanolic and ethanolic crude extracts of E. kebericho against $C$. albicans and $A$. flavus [11].

The activity of E. kebericho essential oil against the fungal strains studied varied with the fungal strain: better activity was observed against $C$. neoformans than the other strains. In a previous study, the essential oil of Lavandula viridis L. and Zingiberaceae species (Z. officinale and Z. cassumunar) showed noteworthy activity against C. neoformans. For C. krusei [30], a comparable result was also observed with the essential oil of Cinnamomum zeylanicum Blume [31]. Higher MIC was observed for C. krusei on control (fluconazole) and available antifungals [32]. A relatively higher MIC was also seen with C. glabrata, although it was less than that seen with C. Krusei. This was in agreement with a study conducted with the essential oil of Artemisia sieberi and Origanum vulgare [33]. Candida krusei is a multidrug-resistant fungal pathogen showing resistance with amphotericin B or fluconazole [34]. A study conducted on the essential oil of Thyme, Summer savory, and Clove against $A$. flavus, however, showed good antifungal activity against $A$. flavus [35]. This difference could be due to differences in the chemical constituents of essential oil between the plants, geographical variations, and/or genetic differences of the isolates used in the study [33].

The essential oil of E. kebericho possessed fungicidal activity against C. glabrata, C. parapsilosis, C. krusei, and
C. neoformans, a finding also seen in a study conducted on Thymus $x$ viciosoi, Angelica major, and Artemisia sieberi essential oil [33, 36, 37]. E. kebericho essential oil, however, showed MFC $>1 \mathrm{mg} / \mathrm{ml}$ against $C$. albicans. In a recent study conducted on Pistacia vera L., the essential oil showed fungicidal effect against $C$. albicans at a concentration greater than $1 \mathrm{mg} / \mathrm{ml}$, a finding which is in agreement with the present study [38]. In another study conducted by Devkatte et al. [39] on essential oils as potential inhibitors of C. albicans growth, out of 38 herbal oils, 9 oils (orange oil, rosemary oil, bergamot oil, clary sage oil, juniper oil, ginger oil, rose oil, Citronella oil, and Eucalyptus oil) also showed higher MFCs [39].

An analysis of the chemical composition of the essential oil of E. kebericho by Hymete et al. revealed sesquiterpenoid compounds as the main component [17]. These compounds could be responsible for the antifungal activity because of their highly lipophilic nature, their low molecular weights, and their capability of disrupting the cell membrane, causing cell death or inhibiting sporulation and germination $[40,41]$.

Two basic methods for the assessment of antimicrobial activities of essential oils are commonly used: the agar diffusion method (paper disc or well) and the broth dilution method. Although the use of microdilution in determining the activity of essential oil is the best way, agar diffusion could be utilized as a screening method, or in combination with a microdilution assay $[2,42]$. In the present study, we used the microdilution method for the determination of MIC and MFC [2, 43]. Most of the antimicrobial molecules in plant extracts are significantly nonpolar and do not diffuse well in the aqueous agar matrix used in agar diffusion. The minimal inhibitory concentration (MIC) method remains the preferred one in the determination of the antimicrobial activity of plant extracts [44].

This study had a number of limitations. First, the study was in vitro and did not reflect the actual therapeutic effect that may arise from variations in pharmacokinetic and pharmacodynamic in vivo models. Second, the volatility of the essential oils could have reduced the activity. Third, the chemical composition of the essential was not studied though it has previously been studied. However, this study provides a broader insight for further in vivo studies that would also include specific experiments involving the isolated and identified active components of the essential oil of E. kebericho.

\section{Conclusion and Recommendations}

In the current study, the essential oil of E. kebericho showed noteworthy activity against C. neoformans and C. albicans. The activity was dose-dependent and fungicidal against C. glabrata, C. neoformans, C. parapsilosis, and $C$. krusei. Further studies are warranted to evaluate the effectiveness of the essential oil in vivo, elaborate the mechanism of action, and isolate and identify the most active component(s). The activity of the combination of the essential oil with conventional antifungal drugs should also be investigated. 


\section{Abbreviations}

ATCC: American type culture collection

CLSI: Clinical laboratory standard institute

DMSO: Dimethyl sulphoxide

MFC: Minimum fungicidal concentration

MIC: Minimum inhibitory concentration

PDA: Potato dextrose agar

RPMI: Roswell park memorial institute medium

SDA: Sabouraud dextrose agar.

\section{Data Availability}

The datasets used and/or analyzed during the current study are available from the corresponding author on request.

\section{Conflicts of Interest}

The authors declare that they have no conflicts of interest.

\section{Authors' Contributions}

TBB, SD, and PEA conceived and developed the research idea and designed the project; TBB and SD collected the plant material and extracted the essential oil; TBB conducted the experiment; PEA mentored and supervised the study process, analyzed data, and provided critical revisions to the manuscript. All authors read and approved the final manuscript.

\section{Acknowledgments}

The authors gratefully acknowledge Addis Ababa University for plant identification and Professor Ariaya Hymete for providing the laboratory for essential oil extraction. Miss Hymanot is gratefully acknowledged for her support during the extraction of essential oil. James Mwesigye from the Microbiology Department at the Faculty of Medicine, Mbarara University, provided assistance in the identification and activity assay of fungal isolates. Lastly, the authors thank the Pharm-Biotechnology and Traditional Medicine Center (Pharm-Biotrac), Mbarara University of Science and Technology, for financial support.

\section{Supplementary Materials}

Supplementary Table: tabular results for linear regression analysis. Supplementary Figure: residual plot for the data. (Supplementary Materials)

\section{References}

[1] Y. Woldeamanuel, "Fungal infection knowledge gap in Ethiopia," The Ethiopian Journal of Health Development (EJHD), vol. 31, pp. 124-126, 2017.

[2] S. Magaldi, S. Mata-Essayag, C. Hartung de Capriles et al., "Well diffusion for antifungal susceptibility testing," International Journal of Infectious Diseases, vol. 8, no. 1, pp. 39-45, 2004.

[3] D. Vázquez-González, A. M. Perusquía-Ortiz, M. Hundeiker, and A. Bonifaz, "Opportunistic yeast infections: candidiasis, cryptococcosis, trichosporonosis and geotrichosis," JDDG: Journal der Deutschen Dermatologischen Gesellschaft, vol. 11, pp. 381-394, 2013.

[4] G. D. Brown, D. W. Denning, N. A. R. Gow, S. M. Levitz, M. G. Netea, and T. C. White, "Hidden killers: human fungal infections," Science Translational Medicine, vol. 4, no. 165, p. 13, Article ID 165rv13, 2012.

[5] D. W. Denning, "Minimizing fungal disease deaths will allow the UNAIDS target of reducing annual AIDS deaths below 500000 by 2020 to be realized," Philosophical Transactions of the Royal Society B: Biological Sciences, vol. 371, 2016.

[6] F. Bongomin, S. Gago, O. Ro, and D. Denning, "Global and multi-national prevalence of fungal diseases-estimate precision," Journal of Fungi, vol. 3, p. 29, 2017.

[7] M. A. Pfaller, "Antifungal drug resistance: mechanisms, epidemiology, and consequences for treatment," The American Journal of Medicine, vol. 125, no. 1, pp. S3-S13, 2012.

[8] T. T. Riley, C. A. Muzny, E. Swiatlo, and D. P. Legendre, "Breaking the mold: a review of mucormycosis and current pharmacological treatment options," Annals of Pharmacotherapy, vol. 50, no. 9, pp. 747-757, 2016.

[9] A. Gómez-López, "Antifungal therapeutic drug monitoring: focus on drugs without a clear recommendation," Clinical Microbiology and Infection, vol. 26, no. 11, pp. 1481-1487, 2020.

[10] T. Teklehaymanot, M. Giday, G. Medhin, and Y. Mekonnen, "Knowledge and use of medicinal plants by people around Debre Libanos monastery in Ethiopia," Journal of Ethnopharmacology, vol. 111, no. 2, pp. 271-283, 2007.

[11] G. Ameya, A. Gure, and E. Dessalegn, "Antimicrobial activity of Echinops kebericho against human pathogenic bacteria and fungi," African Journal of Traditional, Complementary and Alternative Medicines, vol. 13, no. 6, pp. 199-203, 2016.

[12] A. Hymete and A. Kidane, "Screening for anthelmintic activity in two Echinops spp," Ethiopian Pharmaceutical Journal, vol. 9, pp. 67-71, 1991.

[13] A. Debella, A. Taye, D. Abebe, K. Mudi, D. Melaku, and G. Taye, "Screening of some Ethiopian medicinal plants for mosquito larvicidal effects and phytochemical constituents," Pharmacologyonline, vol. 3, pp. 231-243, 2007.

[14] R. Upadhyay, "Essential oils: anti-microbial, antihelminthic, antiviral, anticancer and antiinsect properties," Journal of Applied Biosciences, vol. 36, pp. 1-22, 2010.

[15] F. Nazzaro, F. Fratianni, R. Coppola, and V. D. Feo, "Essential oils and antifungal activity," Pharmaceuticals, vol. 10, no. 4, p. 86, 2017.

[16] A. Mahmoud, R. M. Abd El-Baky, F. Ahmed, and F. M. Gamal, "Antibacterial activity of essential oils and in combination with some standard antimicrobials against different pathogens isolated from some clinical specimens," American Journal of Microbiological Research, vol. 4, pp. 16$25,2016$.

[17] A. Hymete, J. Rohloff, T.-H. Iversen, and H. Kjøsen, "Volatile constituents of the roots of Echinops kebericho Mesfin," Flavour and Fragrance Journal, vol. 22, no. 1, pp. 35-38, 2007.

[18] CLSI, Antifungal Susceptibility Testing of Filamentous Fungi: Approved Standard. CLSI Document M38-A, National Committee for Clinical Laboratory Standards, Wayne, PA, USA, 2002.

[19] A. W. Fothergill, "Antifungal susceptibility testing: clinical laboratory and standards institute (CLSI) methods," in Interactions of Yeasts, Moulds, and Antifungal Agents, pp. 65-74, Springer, Berlin, Germany, 2012. 
[20] CLSI, Method for Antifungal Disk Diffusion Susceptibility Testing of Yeasts: Proposed Guideline (CLSI document M44-A), CLSI, Wayne, PA, USA, 2003.

[21] A. Ingroff, E. Canton, and J. Peman, "Updates in antifungal susceptibility of filamentous fungi," Current Fungal Infection Reports, vol. 3, pp. 133-141, 2009.

[22] Z. C. Gazim, C. M. Rezende, S. R. Fraga, T. I. Svidzinski, and D. A. Cortez, "Antifungal activity of the essential oil from Calendula officinalis L. (asteraceae) growing in Brazil," Brazilian Journal of Microbiology, vol. 39, no. 1, pp. 61-63, 2008.

[23] CLSI, Reference Method for Broth Dilution Antifungal Susceptibility Testing of Yeasts. CLSI Standard M27, Clinical and Laboratory Standards Inistitute, Wayne, PA, USA, 4th edition, 2008.

[24] CLSI, Reference Method for Broth Dilution Antifungal Susceptibility Testing of Filamentous Fungi; Approved Standard. CLSI Document M38-A2, Clinical Laboratory Standards Institute, Wayne, PA, USA, 2008.

[25] E. Pinto, L. Vale-Silva, C. Cavaleiro, and L. Salgueiro, "Antifungal activity of the clove essential oil from Syzygium aromaticum on Candida, Aspergillus and dermatophyte species," Journal of Medical Microbiology, vol. 58, no. 11, pp. 1454-1462, 2009.

[26] L. Imanirampa and P. E. Alele, "Antifungal activity of Cleome gynandra L. aerial parts for topical treatment of Tinea capitis: an in vitro evaluation," BMC Complementary and Alternative Medicine, vol. 16, p. 194, 2016.

[27] K. R. S. França, T. L. Silva, T. A. L. Cardoso, A. L. N. Ugulino, A. P. M. Rodrigues, and A. F. d. M. Júnior, "In vitro effect of essential oil of peppermint (Mentha x piperita L.) on the mycelial growth of Alternaria alternata," Journal of Experimental Agriculture International, vol. 26, no. 5, pp. 1-7, 2018.

[28] T. Arif, J. D. Bhosale, N. Kumar et al., "Natural productsantifungal agents derived from plants," Journal of Asian Natural Products Research, vol. 11, no. 7, pp. 621-638, 2009.

[29] D. Sanglard, "Emerging threats in antifungal-resistant fungal pathogens," Frontiers in Medicine, vol. 3, p. 11, 2016.

[30] I. b. Jantan, M. S. M. Yassin, C. B. Chin, L. L. Chen, and N. L. Sim, "Antifungal activity of the essential oils of nine Zingiberaceae species," Pharmaceutical Biology, vol. 41, no. 5, pp. 392-397, 2008.

[31] M. d. L. Rangel, S. G. d. Aquino, J. M. d. Lima, L. R. Castellano, and R. D. d. Castro, "In vitro effect of Cinnamomum zeylanicum blume essential oil on Candida spp. involved in oral infections," Evidence-Based Complementary and Alternative Medicine, vol. 2018, Article ID 4045013, 13 pages, 2018.

[32] M. A. Pfaller, S. A. Messer, L. Boyken et al., "Use of fluconazole as a surrogate marker to predict susceptibility and resistance to voriconazole among 13,338 clinical isolates of Candida spp. tested by clinical and laboratory standards institute-recommended broth microdilution methods," Journal of Clinical Microbiology, vol. 45, no. 1, pp. 70-75, 2007.

[33] A. R. Khosravi, H. Shokri, S. Kermani, M. Dakhili, M. Madani, and S. Parsa, "Antifungal properties of Artemisia sieberi and Origanum vulgare essential oils against Candida glabrata isolates obtained from patients with Vulvovaginal candidiasis," Journal de Mycologie Médicale, vol. 21, no. 2, pp. 93-99, 2011.

[34] M. A. Pfaller, D. J. Diekema, D. L. Gibbs et al., "Candida krusei, a multidrug-resistant opportunistic fungal pathogen: geographic and temporal trends from the ARTEMIS DISK Antifungal Surveillance Program, 2001 to 2005," Journal of Clinical Microbiology, vol. 46, no. 2, pp. 515-521, 2008.
[35] M. Omidbeygi, M. Barzegar, Z. Hamidi, and H. Naghdibadi, "Antifungal activity of thyme, summer savory and clove essential oils against Aspergillus flavus in liquid medium and tomato paste," Food Control, vol. 18, no. 12, pp. 1518-1523, 2007.

[36] L. Vale-Silva, M. Gonçalves, C. Cavaleiro, L. Salgueiro, and E. Pinto, "Antifungal activity of the essential oil of Thymus $\times$ viciosoi against Candida, Cryptococcus, Aspergillus and dermatophyte species," Planta Medica, vol. 76, no. 09, pp. 882-888, 2010.

[37] C. Cavaleiro, L. Salgueiro, M.-J. Gonçalves, K. Hrimpeng, J. Pinto, and E. Pinto, "Antifungal activity of the essential oil of Angelica major against Candida, Cryptococcus, Aspergillus and dermatophyte species," Journal of Natural Medicines, vol. 69, no. 2, pp. 241-248, 2015.

[38] M. D’Arrigo, C. Bisignano, P. Irrera et al., "In vitro evaluation of the activity of an essential oil from Pistacia vera L. variety Bronte hull against Candida sp." BMC Complementary and Alternative Medicine, vol. 19, no. 1, 2019.

[39] A. N. Devkatte, G. Zore, and S. Karuppayil, "Potential of plant oils as inhibitors of Candida albicans growth," FEMS Yeast Research, vol. 5, no. 9, pp. 867-873, 2005.

[40] J. Tian, X. Ban, H. Zeng, J. He, B. Huang, and Y. Wang, "Chemical composition and antifungal activity of essential oil from Cicuta virosa L. var. latisecta Celak," International Journal of Food Microbiology, vol. 145, no. 2-3, pp. 464-470, 2011.

[41] Y. Hu, J. Zhang, W. Kong, G. Zhao, and M. Yang, "Mechanisms of antifungal and anti-aflatoxigenic properties of essential oil derived from turmeric (Curcuma longa L.) on Aspergillus flavus," Food Chemistry, vol. 220, pp. 1-8, 2017.

[42] D. Kalemba and A. Kunicka, "Antibacterial and antifungal properties of essential oils," Current Medicinal Chemistry, vol. 10, no. 10, pp. 813-829, 2003.

[43] S. Kordali, A. Cakir, H. Ozer, R. Cakmakci, M. Kesdek, and E. Mete, "Antifungal, phytotoxic and insecticidal properties of essential oil isolated from Turkish Origanum acutidens and its three components, carvacrol, thymol and p-cymene," Bioresource Technology, vol. 99, no. 18, pp. 8788-8795, 2008.

[44] J. N. Eloff, "Avoiding pitfalls in determining antimicrobial activity of plant extracts and publishing the results," $B M C$ Complementary and Alternative Medicine, vol. 19, p. 106, 2019. 